\title{
Central motor conduction in brachial monomelic amyotrophy
}

\author{
Pramod K. Pal, Nalini Atchayaram, Gaurav Goel' ${ }^{1}$, Ebenezer Beulah \\ Departments of Neurology and ${ }^{1}$ Neuroimaging and Interventional Radiology, National Institute of Mental Health and \\ Neurosciences, Bangalore, India
}

\begin{abstract}
Background: Prevalence of subclinical involvement of motor pathways in clinically diagnosed Brachial Monomelic Amyotrophy (BMMA) is unknown. Aims: To determine the prevalence of subclinical involvement of central motor pathways in BMMA using transcranial magnetic stimulation. Setting and Design: Prospective case-control study. Materials and Methods: Central motor conduction time (CMCT) was determined by ' $\mathrm{F}$ ' wave method using figure-of-eight coil attached to Magstim 200 stimulator, in 17 patients with BMMA. Motor evoked potentials were recorded from first dorsal interosseous of the affected (AFF) and unaffected upper limbs (UNAFF) at rest and during partial contraction. Comparison was made with data from 10 healthy controls (CTRL). Statistical Analysis: Descriptive analysis and Analysis of Variance (ANOVA). Results: Compared to controls, the mean CMCT of AFF was significantly prolonged, both at rest and contraction: (a) Rest: AFF$6.68 \pm 1.78 \mathrm{~ms}$, UNAFF- $6.36 \pm 1.16 \mathrm{~ms}$, CTRL-5.71 $\pm 1.02 \mathrm{~ms}$; Fisher's PLSD for AFF vs. CTRL: $P=0.037$, (b) Contraction: AFF-5.78 \pm 1.62 $\mathrm{ms}$, UAFF - $4.86 \pm 1.38 \mathrm{~ms}$, CTRL-4.06 $\pm 0.80 \mathrm{~ms}$; Fisher's PLSD for AFF vs. CTRL; $P=0.0002$, AFF vs. UNAFF- $P=0.044$ ). Prolonged CMCT ( $>$ mean +2 SD of controls) was observed in $29.4 \%$ of AFF and $6.25 \%$ of UNAFF at rest, and in $47.1 \%$ and $23.5 \%$ respectively during contraction. Conclusions: Dysfunction of central motor pathways was observed in both affected and unaffected upper limbs of some patients with BMMA of upper limbs. The dysfunction was more pronounced during voluntary contraction. A larger study is needed to validate the significance of these findings.
\end{abstract}

Key words: Central motor conduction time, magnetic stimulation, monomelic amyotrophy.

\section{Introduction}

Transcranial magnetic stimulation (TMS) is a useful tool to study the function of the motor pathways. Abnormalities of central motor conduction time (CMCT) may provide a clue to dysfunction of the corticospinal tracts, even when clinical or magnetic resonance imaging (MRI) may not document such abnormalities.

Monomelic amyotrophy (MMA), is a rare disorder predominantly affecting young men in the second and third decades. In MMA there is segmental anterior horn cell involvement causing wasting and weakness of predominantly one upper or lower limb, without evidence of sensory dysfunction. An upper limb involvement is referred to as brachial MMA (BMMA) or Hirayama disease ${ }^{[1]}$ and a lower limb as crural MMA. ${ }^{[2]}$ There is usually diminution or absence of stretch reflexes of the involved limb and sometimes hyporeflexia may also be present in other limbs, suggesting a more diffuse pathology. In contrast to motor neuron disease, there is usually no clinical evidence of upper motor neuron signs. ${ }^{[3,4]}$ However, brisk reflexes in lower limbs without any other evidence of pyramidal signs are observed in $\mathrm{BMMA}^{[3]}$ and therefore it may be debated whether MMA is a pure entity.

The etiology of Hirayama disease is still poorly understood and possible cord compression or microvascular changes associated with neck flexion have been proposed. ${ }^{[5]}$ Hirayama et al., ${ }^{[6]}$ reported cinematographic MRI showing signal void in posterior epidural space pulsating synchronously with cardiac beat suggesting passive dilatation of the epidural venous plexus. In view of the benign course of the disease, reported autopsies are limited to two patients ${ }^{[7,8]}$ which do not suggest involvement of corticospinal tracts.

Measurement of CMCT may be a useful tool to determine the prevalence of subclinical involvement of motor pathways in patients diagnosed to have MMA and this information may be useful in understanding the pathophysiology as well as prognostication of this disorder. Till date, there are only two studies which have measured CMCT in patients with BMMA, one using transcranial electrical stimulation in seven patients $^{[9]}$ and the other using TMS in 17 patients. ${ }^{[10]}$ Both reported essentially normal CMCT, though there was marginal prolongation in one patient in the

Presented at the XIV Annual Conference of Indian Academy of Neurology, $6^{\text {th }}-8^{\text {th }}$ October, 2006, Bangalore, India. 
former study and two patients in the latter. However, reversible cortico-efferent dysfunction was reported using TMS in a 16-year-old girl with Hirayama disease during sustained neck flexion. ${ }^{[11]}$ None of the above studies have addressed whether the patients with BMMA retain the ability to shorten the CMCT during voluntary contraction of the target muscle, which is normally present in healthy individuals. Therefore we conducted this study to determine whether CMCT of upper limbs is prolonged in MMA both at rest and during partial contraction of the muscle. Comparison was made between the affected and unaffected upper limb and with data from healthy control subjects.

\section{Materials and Methods}

Seventeen consecutive patients (one woman, 16 men) with BMMA were recruited for the study over a period of two years. The criteria for inclusion in the study were a diagnosis of BMMA established clinically and by electoneuromyographic studies to support the diagnosis of pure anterior horn cell involvement in only the affected upper limb and no evidence of conduction block or sensory dysfunction. Magnetic resonance imaging of cervical spine was performed on a 1.5 Tesla machine in 16 patients to rule out cervical myeloradiculopathy.

Data was compared with that from 19 upper limbs of 10 healthy controls, who were working in the hospital. All subjects gave written informed consent. The protocol was approved by the Institute Ethics Committee.

TMS was performed using a figure-of-eight coil attached to a Magstim 200 stimulator (Magstim Company Ltd., Dyfed, United Kingdom). Motor evoked potentials (MEP) were recorded from the first dorsal interosseous (FDI) muscle. The coil was placed on the contralateral motor cortex at the position of best contraction from FDI. The stimulus intensity used was $75 \%$ of maximum stimulator output. MEPs were recorded from FDI of the affected (AFF) and unaffected upper limbs (UNAFF) at rest and during partial contraction. Ten stimuli were applied for each state. The minimum latency (cortex

\begin{tabular}{|c|c|c|}
\hline & Patients & Controls \\
\hline Number & 17 & 10 \\
\hline Women : Men & $1: 16$ & All men \\
\hline $\mathrm{Age}^{*}$ & $\begin{array}{c}23.4 \pm 5.1 \text { years } \\
(16-35)\end{array}$ & $\begin{array}{c}26.7 \pm 2.5 \text { years } \\
(24-30)\end{array}$ \\
\hline Age at onset of symptoms & $\begin{array}{c}19.9 \pm 4.2 \text { years } \\
(13-29 \text { years })\end{array}$ & \\
\hline Duration of symptoms & $\begin{array}{c}46.6 \pm 38.9 \text { months } \\
\text { (10-144 months) }\end{array}$ & \\
\hline \multicolumn{3}{|l|}{ Side affected } \\
\hline Left & $11(64.7 \%)$ & \\
\hline Right & $6(35.3 \%)$ & \\
\hline
\end{tabular}

to muscle) was taken and CMCT, calculated by F wave method. ${ }^{[12]}$

At each state (rest and contraction of the muscle), the mean minimum CMCT for each group (controls, AFF and UNAFF sides) was compared using Analysis of Variance (ANOVA) and the post hoc test used was Fisher's PLSD. In addition, in each patient, the value of CMCT was considered prolonged on the AFF and UNAFF sides if it exceeded the Mean+2SD of the Control group in each state.

\section{Results}

The clinical characteristics of the patients are given in Table 1. The right upper limb was affected (64.7\%) more often than the left (35.3\%). The mean duration of illness, from the time limb wasting was first observed by patient to consultation was around three years.

\section{Clinical findings}

\section{(a) Affected upper limb}

Wasting and weakness predominantly involving small muscles of the hand and forearm were present in all 17 patients. Distal muscle wasting was mild in three (17.6\%), moderate in eight (47.1\%) and severe in six (35.3\%) but weakness was reported in 15 (88.2\%) only. The mean period of progression of illness was $19.3 \pm$ 14.3 months (range $0-48$ ) and the stationary phase at the time of evaluation was $33.5 \pm 37.5$ months $(0-120)$. The other features in the affected limb were presence of cold paresis in 14 (82.4\%), cramps in five (29.4\%), fasciculations in 12 (70.6\%). Muscle tone was normal in $14(82.4 \%)$ reduced in three (17.6\%). Deep tendon reflexes were normal in 10 (58.8\%), sluggish in six (35.3\%) and brisk in one (5.9\%).

Disability secondary to motor weakness in the affected limb was reported by 16 (94.1\%) patients. This was mild in 11 (64.7\%), moderate in four (23.5\%), and severe in one (5.9\%). Ten patients were able to perform all activities of daily living and continue with their jobs. Transfer of skills to the homologous upper limb was reported by five $(29.4 \%)$, and forced change of job by two $(11.8 \%)$ patients.

\section{(b) Contralateral (symptomatically unaffected) upper $\operatorname{limb}$}

Findings in the contralateral upper limb were: asymptomatic mild wasting in five $(29.4 \%)$ with mild weakness in three of them, normal tone in 16 (94.1\%), reduced tone in one (5.9\%), sluggish deep tendon reflexes in 10 (58.8\%), normal deep tendon reflexes in five $(29.4 \%)$ and brisk deep tendon reflexes in two (11.8\%).

\section{(c) Lower limbs}

There were no neurological signs in the lower limbs 


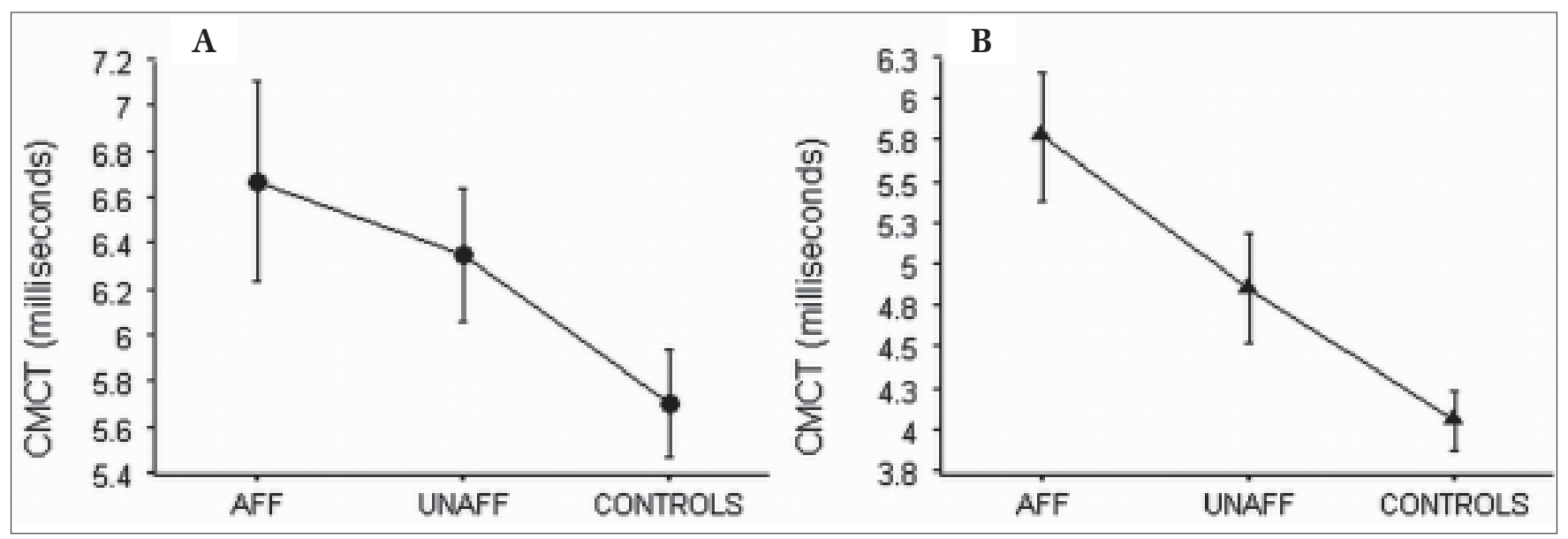

Figure 1: The mean minimum CMCT (milliseconds) are shown (A) at rest and (B) during partial contraction of FDI in affected (AFF) and unaffected (UNAFF) sides of patients and in Controls. At rest ANOVA did not show significant effect of groups $(P=0.09)$, though post hoc test (Fisher's $P L S D)$ showed significant difference between AFF side and control $(P=0.037)$. During contraction ANOVA showed significant effect of groups $(P=0.001)$, and post hoc tests (Fisher's PLSD) showed significant difference between AFF and UNAFF sides $(P=0.044)$ and between AFF side and control $(P=0.0002)$. Error bar=1 SEM

except for brisk reflexes in seven (41.2\%). None had spasticity or positive Babinski's sign.

\section{Electroneuromyography}

Electromyography (EMG) in both upper limbs was performed in all the 17 patients. In the affected limb it was abnormal in all patients. Active denervation was noted in nine (52.9\%), chronic denervation in $15(88.2 \%)$ and reinnervation in 11 (64.7\%). Three patients (17.6\%) had abnormal EMG findings in the contralateral upper limbs: active denervation in one, chronic denervation in three and reinnervation in two. Motor conductions in all the limbs were normal except for reduced amplitude of the compound muscle action potential in six patients with severe wasting of small muscles. There was no evidence of conduction block. Sensory conductions were normal.

\section{Magnetic resonance imaging}

MRI of the cervical spine was performed in 16 of the 17 patients. MRI demonstrated cord atrophy in 12 (75.0\%) of the 16 patients, hemiatrophy in five $(41.6 \%)$ and bilateral cord atrophy in seven (58.3\%). The segments involved were C6 and C7 in five (41.6\%), C5 and C6 in three (25.0\%), from C5 to C7 in three (25.0\%), and C6 in one $(8.3 \%)$ patient. T2 weighted signal changes in the cervical cord were noted in two patients. It involved the anterior C6 and C7 segments symmetrically in one patient and the left lateral column at C5 level in another. Five of these 16 patients underwent neck flexion studies and posterior epidural space was prominently seen in one patient. No demonstrable abnormalities on MRI were observed in four patients.

\section{Transcranial magnetic stimulation findings}

At rest

The mean minimum CMCT at rest in controls was $5.71 \pm 1.02$ ms [Figure 1A]. The CMCT of AFF and UNAFF sides in patients was considered prolonged if it was $>7.75 \mathrm{~ms}$ (Mean+2SD). On the AFF side prolongation was seen in five patients $(29.4 \%)$ and on the UNAFF side in one patient $(6.25 \%)$ [Figure 2A]. The latter patient had normal EMG findings on the unaffected upper limb.

The mean minimum CMCT of the AFF limbs (6.68 $\mathrm{ms}$ ) was greater than the UNAFF limbs (6.36 ms) and both were greater than control $(5.71 \mathrm{~ms})$. ANOVA did not show significant effect of groups $(P=0.09)$, though post hoc test (Fisher's PLSD) showed a significant difference between the AFF side and control $(P=0.037)$ [Figure 1A].

\section{During contraction}

The mean minimum CMCT during contraction in controls was $4.06 \pm 0.80 \mathrm{~ms}$ [Figure 1B]. CMCT of AFF and UNAFF sides in patients was considered prolonged if it was $>5.65 \mathrm{~ms}$ (Mean+2SD). On the AFF side prolongation was seen in eight patients $(47.1 \%)$ and on the UNAFF side in four patients (23.5\%) [Figure 2B]. In the latter group, only one patient had abnormal EMG findings (denervation) on the clinically unaffected upper limb.

The mean minimum CMCT of the AFF limb (5.78 ms) was greater than the UNAFF limb (4.86 ms) and both were greater than control (4.06 ms). ANOVA showed a significant effect of groups $(P=0.001)$, and post hoc tests (Fisher's PLSD) showed a significant difference between the AFF and UNAFF sides $(P=0.044)$ and between AFF side and control $(P=0.0002)$ [Figure 1B]. 


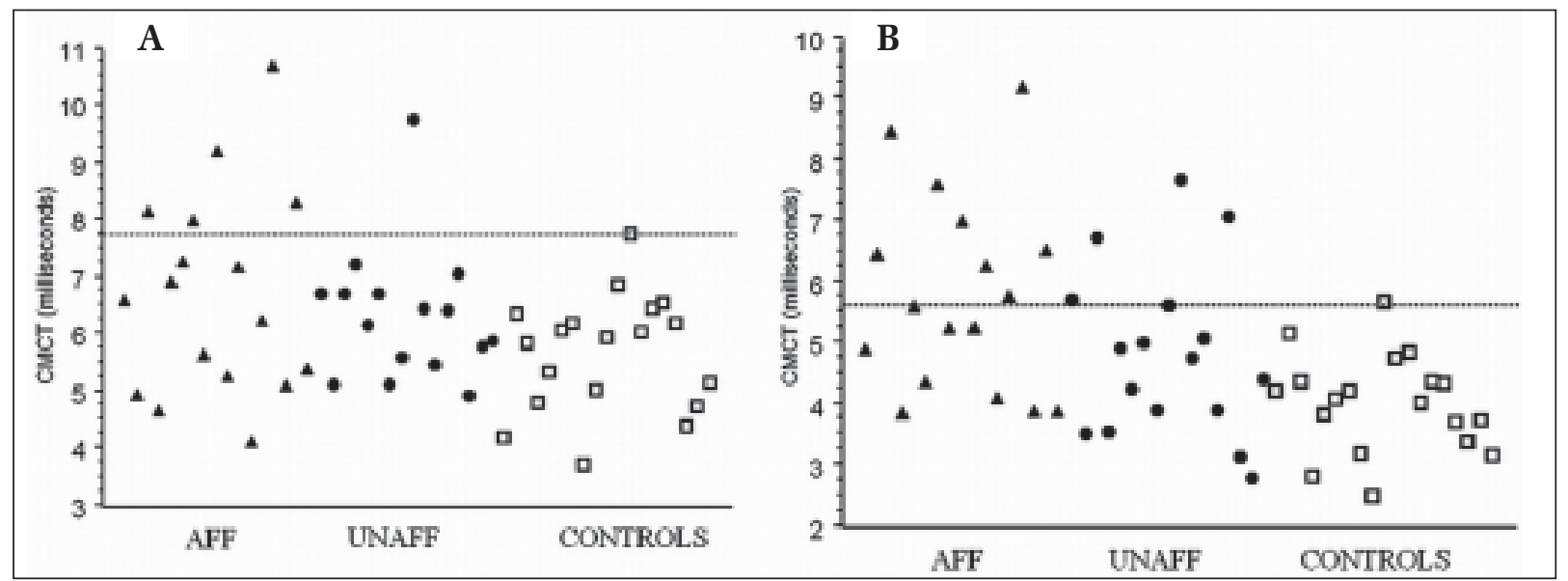

Figure 2: Scatterograms showing the minimum CMCT of each patient on the clinically affected (AFF) and unaffected (UNAFF) upper limbs and in controls $(A)$ at rest and $(B)$ during partial contraction of the first dorsal interosseous muscle. In each figure, the horizontal dotted line represents the Mean+2SD value of the controls, which was $7.75 \mathrm{~ms}$ at rest and $5.65 \mathrm{~ms}$ during contraction. Values above these were considered as prolonged CMCT. CMCT was prolonged in $29.4 \%$ of the AFF and $6.25 \%$ of the UNAFF limbs at rest, and in $47.1 \%$ of the AFF and $23.5 \%$ of the UNAFF limbs during contraction

Comparison between right and left sides in controls

In the controls, based on data from 10 upper limbs of the right side $(\mathrm{R})$ and nine of the left side (L), there was no significant difference between the mean CMCT of the left and right sides both at rest and during contraction [Table 2].

\section{Comparison between CMCT at rest and during contraction}

In controls and both the AFF and UNAFF sides of patients, the mean minimum CMCT during contraction was significantly less than at rest [Table 2], but the degree of significance was much greater in the controls and UNAFF side $(<0.0001)$ compared to the AFF side $(P=0.003)$.

\section{Correlation with duration of illness}

In the affected limbs of the patients, though the duration of illness was longer in those with abnormal CMCT compared to those with normal CMCT, both at rest and during contraction, these differences were not statistically significant (at rest: $55.2 \pm 55.7$ months vs. $43.0 \pm 33.7$ months; during contraction: $64.8 \pm 56.7$ months vs. $39.0 \pm 28.6$ months).

\begin{tabular}{lccc}
\hline \multicolumn{4}{c}{ Table 2: Mean minimum CMCT in controls and patients } \\
\hline & Controls & AFF side & UNAFF side \\
Rest & $5.71 \pm 1.02$ & $6.68 \pm 1.78$ & $6.36 \pm 1.16$ \\
Contraction & $4.06 \pm 0.80$ & $5.78 \pm 1.62$ & $4.86 \pm 1.38$ \\
p $^{*}$ & $<0.0001$ & 0.003 & $<0.0001$ \\
DF $(\mathrm{Cl})$ & $18(1.27-2.03)$ & $16(0.36-1.44)$ & $15(0.93-1.91)$ \\
\hline
\end{tabular}

All values are in milliseconds (ms); *Paired t test; DF (CI): Degree of freedom $(95 \%$ lower-95\% upper confidence interval); In controls, based on data from 10 upper limbs of the right side $(\mathrm{R})$ and nine of the left side $(\mathrm{L})$, there were no significant differences between the sides both at rest (R: $5.40 \pm 0.90, \mathrm{~L}: 6.06 \pm 1.08$ ) and during contraction (R: $4.05 \pm 0.98, \mathrm{~L}: 4.08 \pm 0.59$ ). Based on the data from the 19 upper limbs in controls, the Mean+2SD CMCT was $7.75 \mathrm{~ms}$ at rest and $5.65 \mathrm{~ms}$ during contraction; CMCT-Central motor conduction time, AFF-Affected upper limb, UNAFF-Unaffected upper limb.

\section{Discussion}

Brachial monomelic amyotrophy is thought to be a pure disease of the anterior horn cells. At first clinical examination, it is not uncommon to find a mild asymptomatic wasting of the contralateral upper limb as seen in approximately $30 \%$ of our patients. In general, the course of the illness is benign, and a few studies have looked into the long-term follow-up of these patients. ${ }^{[13,14]}$ Progression of the neurological deficit in the affected limb was seen for up to five years in the majority followed by a stationary phase with no evidence of fresh neurological deficit or appearance of pyramidal signs ${ }^{[13]}$ during the follow-up period.

The pathogenesis of Hirayama disease is unknown and it has been postulated that it may arise from some microcirculatory disturbances in the territory of the anterior spinal artery territory. ${ }^{[15]}$ Apart from causing ischemia of anterior horn cells in the cervical cord, it is also possible that ischemia may involve the adjacent ascending and descending tracts.

It has been often emphasized that pyramidal signs do not appear in BMMA during the course of the illness. ${ }^{[7]}$ However hyperreflexia in lower limbs without Babinski's sign has been commonly reported: in eight of the 20 patients (40\%) reported by Hirayama et al., ${ }^{[4]}$ in three out of seven patients (42.9\%) reported by Misra and Kalitha, ${ }^{[9]}$ in four of 44 patients (9.1\%) by Gouri-Devi and Nalini, ${ }^{[3]}$ and in 58 of 248 patients $(23.4 \%$ ) (Personal communication from one of the co-authors of this paper; the database is from the Department of Neurology, National Institute of Mental Health and Neurosciences, Bangalore, India). In the present study hyperreflexia was seen in 5.9\% of the clinically affected upper limbs, in $12.8 \%$ in the unaffected upper limbs, and in $41.2 \%$ of the lower limbs, but no patient had spasticity, Babinski's sign or 
weakness in pyramidal distribution in any of the limbs. This apparent discrepancy between hypereflexia and absence of other pyramidal signs is difficult to explain. Does hyperreflexia imply a subtle dysfunction of the corticospinal tracts? Is it possible that the corticopsinal pathways that control the stretch reflexes are different from those that control tone, power and plantar reflex and are these more susceptible to injury? Is there any subclinical dysfunction in transmission of neuronal impulse in these pathways, especially during voluntary activities? We tried to address these questions by measuring the CMCT, which reflects the function of the corticospinal tracts. It is known that in healthy subjects CMCT is shortened during voluntary contraction of the target muscle. This facilitation may be a result of preferential activation of the large conducting fibers in the corticospinal tracts. Therefore we measured CMCT both at rest and during partial voluntary contraction of the target muscle to detect any abnormalities of CMCT and to determine whether there is loss of the normal facilitation.

To our knowledge, there are only three published studies $^{[9-11]}$ which have measured CMCT in BMAA, and in all these studies the target muscle was at rest. Shizukawa et al., ${ }^{[11]}$ reported a 16-year-old girl with Hirayama disease who had a mild cervical cord atrophy at the C6 spine level together with an engorged epidural vein at the C4-C6 extradural spaces during neck flexion. TMS during sustained flexion of neck showed a steady attenuation of the amplitude of the evoked potentials from abductor pollicis brevis and the CMCT lengthened as time passed. After $8 \mathrm{~min}$, she began to feel some dullness in the left upper limb. These phenomena were reversible and were observed only on the affected limb. The report supported the presence of a reversible cortico-efferent dysfunction in Hirayama disease and the authors justified the use of neck collars to treat those afflicted with Hirayama disease.

To investigate further if such corticomotor dysfunction exists in BMAA, Misra and Kalita ${ }^{[9]}$ studied CMCT of upper and lower limbs in seven patients with Hirayama disease, using transcranial electrical stimulation. CMCT of lower limbs was normal in all, while that to the upper limb was marginally prolonged in only one patient. Three of their seven patients had lower limb hyperreflexia without any clonus, spasticity or extensor plantar responses. In a later study, the same authors ${ }^{[16]}$ reported that neck flexion did not produce significant changes in N13 and F wave parameters in eight patients with Hirayama disease and was not related to dynamic MRI changes.

In the most recent study, Khandelwal et al., ${ }^{[10]}$ reported cortical threshold intensity and CMCT in 18 patients with MMA. Compared to the controls there was no significant abnormality in cortical threshold intensity and CMCT was normal in all except two patients where it was marginally prolonged. The authors interpreted that this could be because of excessive loss of anterior horn cells.

Our study is not directly comparable to these studies due to difference in methodology (e.g. that by Misra and Kalita $^{[9]}$ had used transcranial electrical stimulation), and we had not measured the cortical motor threshold or the CMCT to lower limbs. Moreover, the previous studies had determined CMCT from the target muscles at rest only.

In healthy subjects, during voluntary contraction of the target muscle, there is activation of the upper motor neuron pool/ activation of faster conducting pyramidal pathways resulting in shortening of the CMCT. Similar to that observed in controls, the CMCT was significantly shortened during contraction of FDI in both the unaffected and affected upper limbs of patients. However, while the degree of the significance on the unaffected side was comparable with the controls, it was less on the affected side. Moreover, the impairment of this facilitation of CMCT was apparent on both the affected and unaffected upper limbs of the patients when compared with the controls. Prolonged CMCT ( $>$ mean +2 SD of controls) at rest was observed in nearly $30 \%$ of the affected upper limbs and in only one on the unaffected side and these abnormalities were more obvious $(47.1 \%$ in the affected upper limb and $23.5 \%$ on the non-affected side) during contraction. Finally, comparison of the mean CMCT values also showed a similar trend: CMCT of affected limb was significantly prolonged compared to controls in both states of FDI, and in addition, during contraction, affected side was significantly more prolonged compared to the unaffected side.

The significance of our findings can only be determined when the patients are followed up to determine if they develop clinical signs of upper motor neuron lesion. The impairment of CMCT facilitation could represent subtle dysfunction of the corticospinal tracts resulting in slowing of impulse propagation, especially during voluntary activity to contract the target muscle. Alternatively, it can signify fallout/ preferential dysfunction of the fast conducting corticospinal fibers, with resultant slower propagation in the remaining slower fibers. Finally, this phenomenon can also be explained by retrograde degeneration of the corticospinal fibers synapsing with the affected anterior horn cells of the C7, C8 and T1 segments.

Our study had several limitations. We did not study the cortical threshold and the CMCT in the lower limbs. Motor threshold has been reported to be normal in previous studies. We used figure-of-eight coil which helped to give a focused stimulation of the cortical representation of FDI muscle. However, technically this coil is unsuitable to cortically stimulate the muscles of the lower limbs. Therefore we did not assess CMCT 
in the lower limbs, though if measured probably would have yielded higher abnormalities (due to long pathways).

Future studies need to focus on assessment of CMCT of both upper and lower limbs, in addition to measurement of cortical motor threshold. Moreover, the progression in BMMA is very slow and the mean duration of symptoms in our patients was less than three years. Therefore larger cohorts with wide range of duration of illness need to be studied to ascertain if there is any positive linear correlation of the CMCT with duration of illness.

\section{Conclusions}

The present study has demonstrated prolongation of central motor conduction time, more evident during voluntary contraction of the target muscle, in both the affected and unaffected upper limbs of some patients with brachial monomelic amyotrophy. This suggests that monomelic amyotrophy may not be a pure anterior horn cell disorder and a subset of patients may have dysfunction of corticospinal pathways.

\section{References}

1. Hirayama K, Toyukura Y, Tsubaki T. Juvenile muscular atrophy of unilateral upper extremity: A new clinical entity. Psychiatr Neurol Jpn 1959;61:2190-7.

2. Gourie-Devi M. Monomelic amyotrophy of upper or lower limbs. In: Wisen AA, Shaw PJ, editors. Handbook of Clinical Neurology. Elsevier, BV; 2007. p. 207-27.

3. Gourie-Devi M, Nalini A. Long-term follow-up of 44 patients with brachial monomelic amyotrophy. Acta Neurol Scand 2003;107:215-20.

4. Hirayama K, Tsubaki T, Toyokura Y, Okinaka S. Juvenile muscular atrophy of unilateral upper extremity. Neurology 1963;13:373-80.
5. Restuccia D, Rubino M, Valeriani M, Mirabella M, Sabatelli M, Tonali P. Cervical cord dysfunction during neck flexion in Hirayama's disease. Neurology 2003;60:1980-3.

6. Hirayama K, Tokumaru Y. Cervical dural sae and spinal cord in juvenile muscular atrophy of distal upper extremity. Neurology 2000;54:1922-6.

7. Hirayama K, Tomonaga M, Kitano K, Yamada T, Kojima S, Arai K. Focal cervical poliopathy causing juvenile muscular atrophy of distal upper extremity: A pathological study. J Neurol Neurosurg Psychiatry 1987;50:285-90.

8. Araki K, Ueda Y, Michinaka C, Takamasu M, Takino T, Konishi H. An autopsy case of juvenile muscular atrophy of unilateral upper extremity (Hirayama's disease). J Jpn Soc Intern Med 1989;78:674-5.

9. Misra UK, Kalita J. Central motor conduction in Hirayama disease. Electroencephalogr Clin Neurophysiol 1995;97:73-6.

10. Khandelwal D, Bhatia M, Singh S, Shukla G, Goyal V, Srivastava T, et al. Threshold intensity and central motor conduction time in patients with monomelic amyotrophy: A transcranial magnetic stimulation evaluation. Electromyogr Clin Neurophysiol 2004;44:357-60.

11. Shizukawa H, Imai T, Kobayashi N, Chiba S, Matsumoto H. Cervical flexion-induced changes of motor evoked potentials by transcranial magnetic stimulation in a patient with Hirayama disease--juvenile muscular atrophy of unilateral upper extremity. Rinsho Shinkeigaku 1994;34:500-3.

12. Mills KR. Brain stimulation in a clinical setting: Magnetic stimulation of the human nervous system. Oxford: Oxford University Press; 1999. p. $168-92$.

13. Gourie-Devi M, Nalini A. Sympathetic skin response in monomelic amyotrophy. Acta Neurol Scand 2001;104:162-6.

14. Rowin J, Meriggioli MN, Cochran EJ. Monomelic amyotrophy with late progression. Neuromuscul Disord 2001;11:305-8.

15. Hirayama K. Non-progressive juvenile spinal muscular atrophy of the distal upper limb (Hirayama disease). In: de Jong JM, editor. Disease of the Motor System. Elsevier Science Publishers BV; 1991. p. 107-20.

16. Misra UK, Kalita J, Mishra VN, Phadke RV, Hadique A. Effect of neck flexion on $\mathrm{F}$ wave, somatosensory evoked potentials, and magnetic resonance imaging in Hirayama disease. J Neurol Neurosurg Psychiatry 2006;77:695-8.

Accepted on 05-08-2008

Source of Support: Nil, Conflict of Interest: None declared. 